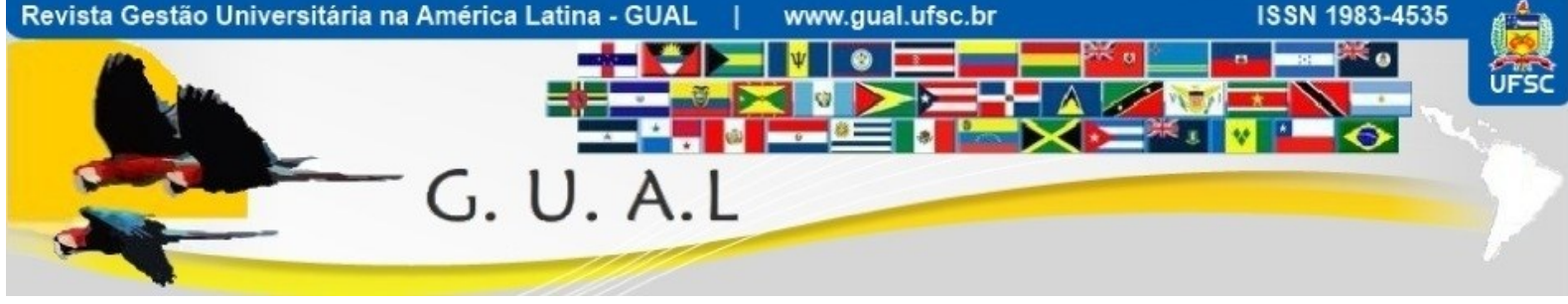

\title{
PLANEJAMENTO ESTRATÉGICO EM UNIVERSIDADES: INOVAÇÃO EM ADMINISTRAÇÃO PÚBLICA ATRAVÉS DE NOVOS MODELOS GERENCIAIS BASEADOS EM SISTEMAS DE INFORMAÇÃO
}

\author{
STRATEGIC PLANNING IN UNIVERSITIES: INNOVATION IN PUBLIC \\ ADMINISTRATION THROUGH NEW MANAGEMENT MODELS BASED ON \\ INFORMATION SYSTEMS
}

Joni de Almeida Amorim, PhD Universidade Estadual de Campinas - UNICAMP joni.amorim@gmail.com

Juliano Schimiguel, PhD Universidade Cruzeiro do Sul - UNICSUL schimiguel@gmail.com

Recebido em 12/março/2012

Aprovado em 30/julho/2012

Sistema de Avaliação: Double Blind Review

Esta obra está sob uma Licença Creative Commons Atribuição-Uso. 


\begin{abstract}
RESUMO
Hoje se percebe uma necessidade de melhoria do planejamento estratégico das universidades, em especial pela necessidade de que se tenha como foco a crescente competição entre as Universidades de Classe Mundial, pelos recursos e pelas posições nos mais variados rankings nacionais e internacionais hoje existentes. Nessa perspectiva, este texto tem como objetivo discutir o planejamento estratégico universitário considerando o caso especial da UNICAMP, hoje vista como uma das mas melhores universidades brasileiras. $\mathrm{O}$ texto apresenta uma visão geral da temática, apresenta o caso da UNICAMP e do seu sistema de informação em uso, apresenta as necessidades de um novo modelo gerencial e, por fim, propõe um novo modelo que busca abarcar as características hoje vistas como essenciais em sistemas voltados ao planejamento estratégico. O texto também discute tendências futuras que tendem a afetar a realidade das Universidades de Classe Mundial no tocante a sistemas de informação voltados ao planejamento estratégico.
\end{abstract}

Palavras-chave: Administração pública. Competitividade. Internacionalização. Planejamento.

\begin{abstract}
Today there is a need for improvement on the strategic planning in universities, in particular the need to consider the growing competition between World Class Universities for resources and positions in any of the various national and international rankings that exist today. In this perspective, this paper aims to discuss strategic planning in universities while considering the special case of UNICAMP, now seen as but one of the best universities in Brazil. The paper presents an overview of the subject, presents the case of UNICAMP and its information system in use, presents the needs of a new management model and, finally, proposes a new model that seeks to encompass the features now seen as essential to systems dedicated to strategic planning. The text also discusses future trends that tend to affect the reality of World Class Universities in relation to information systems aimed at strategic planning.
\end{abstract}

Keywords: Public administration. Competitiveness. Internationalization. Planning. 


\section{INTRODUÇÃO}

A Universidade Estadual de Campinas (UNICAMP) promoveu de 18 a 21 de julho de 2012 a 1" Escola "Zeferino Vaz” de Educação Superior (http://www.gr.unicamp.br/ceav/ezv/). Tal evento, que contou com a participação do primeiro autor deste texto, visou propiciar aos dirigentes do ensino superior a oportunidade de interagir com especialistas e pesquisadores de renome no Brasil e no exterior. Os temas incluíram internacionalização, desenvolvimento da cultura acadêmica, inovações em sala de aula e permanência e sucesso acadêmico. Dentre os muitos conferencistas convidados, podem ser destacados Philip Altbach, Liz Reisberg e Karen Arnold, do Boston College (EUA), Philip Baty, editor do Times Higher Education (Reino Unido), Peter Dourmashkin, do Massachussetts Institute of Technology (EUA), Pedro Pablo Rosso, da Universidade Católica de Santiago (Chile), Jamil Salmi, ex-Coordenador de Educação Superior do Banco Mundial, e Simon Schwartzman, do Instituto para Educação e Trabalho (Brasil).

Dentre as muitas conclusões do evento citado, pode-se incluir a necessidade de melhoria da administração das universidades, o que inclui o planejamento estratégico. Merece destaque a necessidade de que se tenha como foco a crescente competição entre as Universidades de Classe Mundial, ou World Class Universities (WCUs). Essa competição inclui desde os recursos financeiros e humanos até a busca por melhores posições nos mais variados rankings nacionais e internacionais hoje existentes.

Nessa perspectiva, este texto tem como objetivo discutir o planejamento estratégico universitário considerando o caso especial da UNICAMP, hoje vista como uma das melhores universidades brasileiras e, mais ainda, uma das melhores do mundo dentre as que possuem menos de cinquenta anos de existência pelo novo ranking da Times Higher Education (THE http://europe.nxtbook.com/nxteu/ts1/100under50/index.php). O texto apresenta uma visão geral da temática, apresenta o caso da UNICAMP e do seu sistema de informação em uso para o planejamento estratégico, apresenta as necessidades de um novo modelo gerencial e, por fim, propõe um novo modelo que busca abarcar as características hoje vistas como essenciais em sistemas voltados ao planejamento. O texto também discute tendências futuras que tendem a afetar a realidade das WCUs no tocante a sistemas de informação voltados ao planejamento estratégico. 


\section{PLANEJAMENTO ESTRATÉGICO}

A missão de uma organização é seu propósito de longo prazo. Objetivos são alvos específicos e mensuráveis que a organização pode utilizar para avaliar até que ponto está realizando a sua missão. Bons objetivos são fáceis de medir e de acompanhar ao longo do tempo. Definidos os objetivos, fazemos as análises interna (forças e fraquezas) e externa (ameaças e oportunidades). Depois, fazemos as escolhas estratégicas.

O planejamento é como um mapa a ser seguido pelas pessoas em suas atividades futuras, para que consigam atingir os objetivos da organização, seja pública ou privada. Temos no mínimo três níveis de planejamento: (1) alto, com gerentes de alto nível, ou gerentes estratégicos; (2) médio, com gerentes de nível intermediário, ou gerentes táticos; e (3) baixo, com gerentes da linha de frente, ou gerentes operacionais. O planejamento estratégico considera a tomada de decisões sobre os objetivos e as estratégias de longo prazo, como planejar uma fusão ou uma aquisição. O planejamento tático traduz o planejamento estratégico (muito amplo e genérico) em metas e planos específicos para uma parte da organização como, por exemplo, planos de marketing, de recursos humanos etc. O planejamento operacional considera períodos de tempo bastante curtos, com foco em atividades rotineiras, como a realização de entregas de produtos, a venda diária de produtos por comércio eletrônico, a interação com os clientes etc.

Desde 1959 já se sabia da importância de se estudar planejamento estratégico inclusive na graduação, conforme o relatório de 494 páginas feito na época (GORDON; HOWELL, 1959). Até hoje tal relatório é discutido por publicações relevantes como a revista "The Economist" (THE GORDON-HOWELL, 2009, p. 1), que destacou em 2009 que o relatório foi escrito por uma dupla de economistas, Robert Aaron Gordon e James Edwin Howell, com apoio da Fundação Ford. Tal texto chocou as escolas da época, dominando a revisão dos currículos nos anos seguintes e sugerindo, por exemplo, que os docentes das escolas de administração se dedicassem mais à pesquisa e menos à consultoria. Inicialmente, como explicam Certo e Peter (2005, p. 3), o objetivo do relatório era o de que se inclú́sse uma disciplina abrangente de "política de negócios", que buscasse enfatizar identificação, análise e solução de problemas do mundo real de maneira ampla, com o desenvolvimento de habilidades importantes para a futura atuação profissional. Ainda que em momentos anteriores a disciplina de planejamento estratégico fosse obrigatória apenas para alunos de 
graduação em administração, hoje já se percebe sua importância em inúmeras áreas, inclusive em áreas como engenharia.

Kiechel (2011, p. 11) destaca que as consultorias contribuíram para a intelectualização dos negócios, tornando a estratégia um alicerce fundamental para o pensamento administrativo. $\mathrm{O}$ autor registra a ascensão do setor de consultoria relacionada ao planejamento estratégico: mais de cinco bilhões de dólares anuais em todo o mundo, principalmente para grandes organizações. Mas o planejamento estratégico também é importante para as micro e as pequenas empresas, sendo visto por alguns estudos (SEBRAE, 2004, p. 26) como o tema mais essencial nos primeiros doze meses de funcionamento de um empreendimento.

Conforme indicam Mintzberg, Ahlstrand e Lampel (2010, p. 20), há dez escolas de pensamento sobre o planejamento estratégico e nenhuma delas é suficientemente completa. $\mathrm{O}$ caso da fábula "Os Cegos e o Elefante", citada pelos autores, exemplifica bem o problema: sem ver o animal completo, cada cego dá uma descrição parcial e/ou incompleta do elefante, da mesma forma que os teóricos da administração dão uma descrição incompleta do planejamento estratégico. Os autores destacam que, das dez escolas, as três primeiras seriam prescritivas, ou mais preocupadas em como as estratégias devem ser formuladas ("prescritas"): Escola do Design, Escola do Planejamento e Escola do Posicionamento. Depois, teríamos ainda sete escolas descritivas, focadas em descrever como as estratégias são formuladas, enquanto a décima escola seria uma combinação de várias outras. Os autores ainda salientam que existem inúmeros modelos de planejamento estratégico, mas que a maioria se reduz às mesmas ideias básicas.

Das dez escolas, a Escola do Design é a mais influente do processo de formulação da estratégia, cabendo destacar a famosa noção de SWOT (MINTZBERG; AHLSTRAND; LAMPEL, 2010, p. 36), uma sigla para Forças (Strengths), Fraquezas (Weaknesses), Oportunidades (Opportunities) e Ameaças (Threats). Tal escola apresenta sete premissas essenciais, como a de que o modelo de formulação da estratégia deve ser mantido simples e informal. Dentre as muitas críticas está o fato de que muitas vezes os pontos fortes e os pontos fracos são insatisfatoriamente determinados.

$\mathrm{Na}$ Escola do Planejamento, por sua vez, a formulação de estratégia se torna um processo formal, com o foco na maior formalização para se ter maior controle. É criado um sistema (MINTZBERG; AHLSTRAND; LAMPEL, 2010, p. 63) com vários planos que se 
completam, para permitir o controle. Tal escola originou-se com Ansoff, em 1965, quase ao mesmo tempo em que a Escola de Design. Resumidamente, ela pretendia que a estratégia fosse guiada por um quadro de planejadores altamente educados dentro da organização, com um departamento especializado em planejamento estratégico, com procedimentos, treinamentos e análises formais, além de muitos números.

As três premissas essenciais da Escola do Planejamento são (MINTZBERG; AHLSTRAND; LAMPEL, 2010, p. 67): foco no planejamento formal; uma equipe de planejadores que se responsabiliza pela execução; e estratégias que surgem prontas desse processo, com atenção detalhada a objetivos, orçamentos, programas e planos operacionais de vários tipos. Ou seja, nessa escola temos muito formalismo e muitos detalhes. Um dos maiores problemas é a inflexibilidade dos "planos" feitos, pois isso dificulta adaptações ao ambiente externo em constante mudança.

Um dos avanços da Escola do Planejamento é o planejamento de cenários (SHOEMAKER, 1995, p. 26). Nesse caso, o cenário baseia-se na suposição de que o futuro não é previsível, mas é possível especular sobre uma variedade de futuros analisando vários cenários. O interesse pela ferramenta teve início com Wack em 1985, em um artigo onde se descrevia a construção de cenários na Shell antecipando o drástico aumento de preços do petróleo em 1973. O planejamento por cenários é um método disciplinado para que futuros possíveis sejam imaginados, o que permite às organizações gerar e avaliar opções estratégicas. A técnica pode ser aplicada a virtualmente qualquer situação na qual o tomador de decisão queira imaginar como o futuro poderia ser.

No planejamento estratégico, as estratégias podem ser chamadas de deliberadas quando a estratégia pretendida originalmente é realizada - e de emergentes - quando a estratégia realizada não era pretendida. No mundo real, as estratégias são uma mistura entre os dois tipos (MINTZBERG; AHLSTRAND; LAMPEL, 2010, p. 26).

Supondo que a organização e/ou que uma unidade sua já formulou uma estratégia, como implementá-la? Ou seja, se foram feitas as escolhas estratégicas, como implementar a estratégia? Como escolher e elaborar indicadores? Como desenvolver o plano de implementação? É possível fazer tudo isso na perspectiva do Balanced Scorecard (BSC), uma proposta de sistema gerencial muito usada, inclusive em universidades como a UNICAMP. Tal sistema foi originalmente desenvolvido pelo engenheiro com doutorado em pesquisa operacional e professor da Harvard Business School Robert S. Kaplan, em conjunto com 
David P. Norton, também engenheiro com pós-graduação em pesquisa operacional e doutorado em administração.

Kaplan e Norton (1997, p. 7) acreditam que o modelo da contabilidade financeira deve se ampliar de modo a incorporar a avaliação dos ativos intangíveis e intelectuais de uma organização pública ou privada, como produtos e serviços de alta qualidade, funcionários motivados e habilitados, processos internos eficientes e consistentes e, mais ainda, clientes satisfeitos e fiéis. Em resumo, eles advogam que é um erro focar apenas em medidas financeiras do desempenho passado; com isso, o BSC busca complementar as medidas financeiras. Cada objetivo estratégico será associado a uma das quatro perspectivas do BSC: (1) Perspectiva financeira; (2) Perspectiva dos clientes; (3) Perspectiva dos processos Internos; e (4) Perspectiva de aprendizagem e crescimento. Os indicadores estratégicos são associados aos objetivos estratégicos definidos originalmente. Assim, coletamos dados localmente onde as pessoas trabalham no dia a dia, transformamos tais dados em informações e, em seguida, atualizamos nossos indicadores estratégicos.

Kaplan e Norton (1997, p. 3) advogam que estamos na era da informação e não mais na era industrial (que transcorreu de 1850 a 1975). Assim, com o advento da era da informação, muitas das premissas fundamentais da concorrência industrial tornaram-se obsoletas. Em poucas palavras, a geração de valor futuro deve direcionar os investimentos em clientes, fornecedores, funcionários, processos, tecnologia e inovação. O BSC preserva o interesse no desempenho de curto prazo com a perspectiva financeira, mas também releva os vetores de valor para um desempenho financeiro e competitivo superior a longo prazo. Com isso, o BSC leva o conjunto de objetivos de cada uma das unidades de uma organização além das medidas financeiras sumarizadas. As quatro perspectivas permitem, portanto, um equilíbrio (balanceamento) entre indicadores que traduzem a missão e a estratégia como um todo.

O BSC traduz a missão e a estratégia de uma unidade da organização em objetivos e medidas tangíveis. Desse modo, pretende-se viabilizar quatro processos gerenciais críticos pelo uso do BSC (KAPLAN; NORTON, 1997, p. 9): (1) esclarecer e traduzir a visão e a estratégia; (2) comunicar e associar objetivos e medidas estratégicas; (3) planejar, estabelecer metas e alinhar iniciativas estratégicas; e (4) melhorar o feedback e o aprendizado estratégico. Assim, definimos os objetivos (objectives) da organização, para depois definir os indicadores e as medidas (indicators/measures) com suas respectivas metas (targets) que serão atingidas 
através de iniciativas (initiatives). O BSC cria uma estrutura e uma linguagem para comunicar a missão e a estratégia, utilizando indicadores para informar os funcionários sobre os vetores do sucesso atual e futuro. Ele deve ser utilizado como sistema de comunicação, informação e aprendizado, e não como um sistema de controle (KAPLAN; NORTON, 1997, p. 25).

Quando já existir consenso sobre a importância e a relevância do BSC, deve-se iniciar uma busca por maior clareza no entendimento da estratégia da organização como um todo e de cada unidade em separado (KAPLAN; NORTON, 1997, p. 309). Em uma organização pouco diversificada, as estratégias das unidades devem coincidir com a estratégia da organização. Em uma organização muito diversificada, será necessário que cada unidade compreenda bem a estratégia da organização antes de elaborar a sua, pois esta tende a não coincidir com aquela. O BSC da organização deve refletir a estratégia corporativa e articular os motivos para a existência de muitas ou poucas unidades operando de maneira mais ou menos independente dentro de tal organização. Desse modo, o BSC da organização vai ser compreendido pelas unidades que depois gerarão o seu BSC específico.

O BSC da organização deve buscar esclarecer dois elementos da estratégia (KAPLAN; NORTON, 1997, p. 178): (1) temas corporativos, como valores, crenças e temas que refletem a identidade corporativa e devem ser compartilhados por todas as unidades; e (2) o papel corporativo, como ações impostas em nível corporativo que criam sinergias no nível das unidades. Assim, os BSCs das unidades podem ser pouco ou muito personalizados, mas todos devem ter seu foco e seu propósito derivados do BSC corporativo.

Kaplan e Norton (1997, p. 320) sugerem as seguintes medidas essenciais de resultados como sendo as que aparecem com mais frequência em BSCs. Para os indicadores financeiros essenciais teríamos, por exemplo, os seguintes: (1a) retorno sobre o investimento; (1b) lucratividades; (1c) aumento de receita; e (1d) produtividade e/ou redução de custos. Para as medidas essenciais dos clientes, poderíamos ter: (2a) participação de mercado; (2b) aquisição de novos clientes; (2c) retenção de clientes atuais; (2d) lucratividade por tipo de cliente; e (2e) satisfação dos clientes. No caso das medidas essenciais de aprendizado e crescimento, incluem-se: (3a) satisfação dos funcionários; (3b) retenção de funcionários; e (3c) lucratividade por funcionário. Por fim, para a perspectiva de processos internos, os autores não sugerem as medidas essenciais de resultados que aparecem com mais frequência em BSCs, sendo possível, nesse caso, analisar as medidas para as demais três perspectivas e depois definir os indicadores dessa quarta perspectiva com foco em viabilizar o que se 
pretende realizar para a unidade como um todo, dado que isso depende dos processos internos.

Pesquisas recentes (http://www.thepalladiumgroup.com) indicam que o uso combinado de BSC e de softwares de "Business Intelligence" (BI), de maneira geral, produz resultados muito melhores que o uso em separado de BSC. BI se refere ao processo de buscar usar informática no apoio à coleta de informações, assim como ao de armazenar e analisar o que foi coletado para então realizar a tomada de decisão. Em administração estratégica, BI pode nos auxiliar a organizar dados, gerando informações que depois se transformam em conhecimento útil: dados puros são transformados em informação relevante através de ferramentas e técnicas diversas. Os softwares para BI nos auxiliam, portanto, a utilizar melhor os dados disponíveis. Alguns desses dados vão alimentar indicadores úteis ao BSC. Nesse sentido, Kaplan e Norton (1997, p. 322) sugerem que o plano de implementação do BSC inclua a identificação de como os indicadores de interesse se ligam aos bancos de dados e aos sistemas de informações, comunicando o BSC e permitindo o desenvolvimento de métricas que depois se evidenciam em "dashboards".

Assim, no BSC, os dados são utilizados para gerar indicadores de performance que permitam verificar se a estratégia está sendo efetivamente implementada nas unidades da organização. O uso crescente de softwares de "Business Intelligence" (BI) é apenas uma das muitas evidências do crescimento da cultura orientada a "analytics", que se refere ao uso de dados e de introspecções associadas a eles com o apoio de disciplinas como estatística, ciências cognitivas, matemática aplicada e outras. O uso de "analytics" (KIRON et al., 2011, p. 17) permite basear em fatos desde o planejamento até as decisões, passando pela execução, pelo gerenciamento e pela medição, incluindo por fim a aprendizagem organizacional. De maneira simplificada, "analytics" pode ser útil para: (a) análise descritiva, para se entender o que aconteceu, como, por exemplo, para ver dados na produção na fábrica e monitorar indicadores; (b) análise preditiva, para tentar prever o que pode acontecer, como, por exemplo, para ver dados sobre aumento das vendas e extrapolar utilizando gráficos etc.; e (c) análise prescritiva, sugerindo o que seria o mais correto e que deveria acontecer, como, por exemplo, com sugestões de como melhorar processos.

Em uma cultura orientada a "analytics", ferramentas e técnicas para o que se chama de "big data" podem vir a ser de interesse, o que nesse caso inclui novos modos de agregar, manipular, analisar e visualizar grandes volumes de dados em uma abordagem flexível e 
multidisciplinar (MGI, 2011, p. 15). Exemplos de ferramentas e técnicas incluiriam: (a) "crowdsourcing", uma técnica para coletar dados submetidos por uma comunidade ou por um grupo de pessoas através de redes como a Web 2.0; (b) "predictive modeling", um conjunto de técnicas no qual um modelo matemático é criado ou escolhido para melhor prever a probabilidade de um evento; (c) "simulation", modelando o comportamento de sistemas complexos para previsão (“forecasting”), predição (“predicting”) e planejamento por cenários ("scenario planning"); e (d) "visualization", técnicas utilizadas para criar imagens, diagramas ou animações para comunicar, entender e melhorar os resultados de análises de grandes volumes de dados.

Percebe-se, portanto, que o plano de implementação do BSC deve incluir a identificação de como os indicadores de interesse se ligam aos bancos de dados e aos sistemas de informações. Com isso, o uso de ferramentas e técnicas diversas que promovam a automação parcial ou total de processos relevantes deve estar associado ao planejamento estratégico, como a coleta de dados relevantes a indicadores que, por sua vez, são visualizados em "dashboards". Essa evidente complexidade hoje associada ao BSC fez com que seus criadores, Kaplan e Norton, desenvolvessem um novo conceito: o de "Office of Strategic Management" (OSM).

OSM (KAPLAN; NORTON, 2008, p. 289) se refere a uma unidade da organização que presta suporte ao planejamento, com foco na excelência na execução operacional. A proposta de OSM surgiu da observação pelos autores de que o desempenho não era sustentável após a implantação do BSC em muitas das organizações, pois estas baseavam seu sucesso inicial apenas em uma liderança forte. Os autores notaram que o desempenho só era sustentável nas organizações em que um pequeno número de gestores, pouco numeroso, mas dedicado, tinha a função de supervisionar os vários processos necessários à execução da estratégia. Tal grupo foi chamado, então, de OSM. Confrontando teoria e prática, os autores identificaram todos os processos fundamentais necessários à implementação de um princípio muito importante: converter a estratégia em um processo contínuo. Pretende-se assim que as organizações passem a construir fortes vínculos entre estratégias e operações, para que as atividades operacionais do dia a dia efetivamente promovam os objetivos estratégicos.

Com base em suas descobertas mais recentes, Kaplan e Norton integram os resultados de seus quatro livros anteriores e criam um novo sistema gerencial autônomo e abrangente de “loop" fechado (2008, p. 290). É um ciclo, portanto, com seis estágios que buscam unir os 
métodos e as práticas desenvolvidos em torno dos livros sobre o BSC. O OSM ficaria responsável pelos novos processos relacionados aos estágios do ciclo. $\mathrm{O}$ escritório tem três funções importantes: (a) arquitetar o planejamento estratégico, o que envolve projetar (criar) novos processos de gestão da estratégia e da operação; (b) integrar e sincronizar os diversos processos da organização que se interliguem com a estratégia, como gestão de recursos humanos (HR), gerenciamento de projetos (PMO), gestão de recursos financeiros, gestão de tecnologia de informação (IT) etc.; e (c) ser dono dos processos necessários à gestão da estratégia, inclusive executando tais processos que transcendem fronteiras das unidades, os quais aparecem no ciclo de seis estágios do sistema gerencial proposto.

A seção seguinte apresenta o planejamento estratégico em uma universidade brasileira e discute a necessidade de que ocorra uma transformação na cultura organizacional para que se tenha sucesso na utilização de métodos e práticas baseados em soluções como BSC e OSM. Discute-se, desse modo, a necessidade de mudança na forma de gerenciar.

\section{PLANEJAMENTO NA UNIVERSIDADE ESTADUAL DE CAMPINAS}

As razões para o planejamento em uma universidade pública como a UNICAMP podem incluir: efetividade organizacional; atribuição apropriada de responsabilidade advinda de regulamentações estabelecidas pelo governo e pela sociedade; comprometimento e engajamento tanto com a comunidade interna quanto com a comunidade externa; clarificação do propósito e das prioridades da universidade; e reconhecimento da identidade da universidade.

A UNICAMP é uma das poucas universidades brasileiras a ter um planejamento estratégico estruturado, motivo pelo qual se tornou referência no assunto dentro e fora do Brasil. A sua metodologia foi apresentada em países como Equador, República Dominicana, Canadá e Turquia e serve de "benchmarking” (GARDENAL, 2008) para outras instituições de ensino superior que buscam a maximização do seu desempenho. Tal metodologia, que começou a ser utilizada de maneira mais sistemática a partir de 2002 com a sensibilização da comunidade, viabiliza a reavaliação periódica de ações e projetos estratégicos em andamento ou já planejados.

Assim, o Planejamento Estratégico da Universidade Estadual de Campinas (PLANESUNICAMP - http://www.cgu.unicamp.br/pei/) é um processo acadêmico e administrativo que busca o estabelecimento de prioridades de médio e longo prazo, ao mesmo tempo em que 
favorece a formulação e a implantação de políticas que levem à realização dessas prioridades. Segundo Barbieri (2008), o PLANES foi idealizado pelo engenheiro eletrônico Eliezer Arantes da Costa, o qual defendeu sua tese de doutorado na Faculdade de Engenharia Elétrica e de Computação (FEEC) em abril de 2008. O PLANES evidencia a missão, os princípios, os valores e a visão de futuro da Universidade, em cinco áreas estratégicas (UNICAMP, 2008, p. 3): (a) ensino, (b) pesquisa, (c) extensão e cooperação técnico-científica e cultural, (d) administração e gestão e (e) qualidade de vida.

Para que universidades como UNICAMP tenham sucesso na utilização de métodos e práticas baseados em soluções como BSC e OSM, se faz necessário que ocorra uma transformação na cultura organizacional que leve a uma mudança na forma de gerenciar. Realizar uma transição é ir de um estado inicial A para um estado final B. Alguns exemplos de mudanças são os seguintes: (1) uma organização ainda pouco informatizada precisa passar a ter sistemas integrados de gestão, ou "Enterprise Resource Planning” (ERP) pois seus concorrentes já o fazem e são mais eficientes; (2) uma organização precisa passar a considerar a sustentabilidade ambiental pois os consumidores só querem produtos que não destruam a natureza etc.; (3) uma organização precisa passar utilizar algum sistema de planejamento melhor, como o BSC, pois atualmente os empregados não participam.

O problema das transições é que nem todos querem mudar. Trata-se da resistência à mudança, pois as pessoas geralmente não querem fazer a transição de um estado para outro, já que isso envolve aprender novas formas de trabalhar etc. Para lidar com isso, podem ser utilizadas estratégias de gestão da mudança (transição), ou "Management of Change" (MoC). Exemplificando esse conceito, Kotter (1996) sugere as seguintes atividades essenciais para que um gestor lidere a mudança: (1) estabeleça um senso de urgência; (2) junte um grupo com poder para the ajudar; (3) crie uma visão de qual será o contexto após a mudança; (4) comunique (explique) a visão a todos; (5) motive as pessoas a ajudar na mudança; (6) mostre cada pequena vitória; (7) promova novas mudanças; e (8) fixe a mudança na cultura.

Ao discutir a gestão da mudança (MoC), Harrington, Conner e Horney (1999, p. 100) definem "Business Process Improvement" (BPI), ou Melhoria dos Processos de Negócios, como um conjunto de metodologias utilizadas para melhorar processos, o que inclui redesenho de processos, reengenharia de processos, "benchmarking", reestruturação e projetos de implantação de softwares como ERP. Tais autores registram que MoC não tem como foco o que será mudado, como por exemplo a solução de BPI, mas sim como a solução 
será implementada, desse modo tendo como objetivo aumentar substancialmente as chances de sucesso do projeto pela consideração de aspectos sociais relacionados à mudança. Nessa perspectiva, alguns dos processos de MoC seriam: a construção da arquitetura da implementação; o gerenciamento da resistência individual à mudança; a construção de comprometimento individual com a mudança; o gerenciamento dos aspectos culturais da mudança; a seleção e a entrega de agentes de mudança. Algumas ferramentas de MoC seriam: formulário de descrição do projeto de mudança; previsão do impacto da mudança; pesquisa histórica de mudanças; ferramenta de mapeamento; plano de anúncio de mudança; avaliação de patrocinadores; pesquisa de cenários; escala de resistência à mudança; avaliação de agentes de mudança; e plano de implementação de mudança organizacional.

Harrington, Conner e Horney (1999, p. 155) explicitam sete estágios principais pelos quais passam as pessoas quando estas percebem uma mudança como negativa: (1) imobilização; (2) negação; (3) raiva; (4) negociação; (5) depressão; (6) teste; e (7) aceitação. Por outro lado, quando percebem a mudança como positiva, as pessoas passam por cinco estágios: (1) otimismo desinformado, ou certeza; (2) pessimismo informado, ou dúvida; (3) realismo esperançoso; (4) otimismo informado, ou confiança; e (5) finalização, ou satisfação. Tais autores salientam que muitos não completam os ciclos citados, repetindo-os em parte ou totalmente, sendo que aqueles que completam a transição respondem a ela com diferentes ritmos intelectuais e emocionais. Nesse contexto, torna-se fundamental ao gerente do projeto compreender que o aumento da resiliência da equipe, assim como a minimização da chance de ocorrer comportamento disfuncional, depende do grau com que tal gerente compreende a cultura organizacional, desse modo evitando discrepâncias entre a cultura corrente e os objetivos do projeto. Com isso, deve-se buscar uma arquitetura de implementação de MoC com as seguintes fases: (1) esclarecer o escopo do projeto; (2) anunciar o projeto; (3) conduzir o diagnóstico; (4) desenvolver um plano de implementação; (5) executar o plano; (6) monitorar o progresso e os problemas; e (7) avaliar os resultados finais.

Como é evidente, uma mudança organizacional pode ser entendida como uma diferença em forma, qualidade ou estado ao longo do tempo em uma organização. Uma mudança afeta uma entidade tal como um indivíduo, um grupo de indivíduos, uma organização ou um conjunto de organizações, com a mudança sendo detectada através de algum tipo de medição realizada em dois ou mais momentos ao longo do tempo, de modo que comparações possam ser feitas entre um estado inicial e um estado final. A literatura sobre 
mudança organizacional discute a natureza da diferença entre um estado e outro, o que produziu a transição e quais as suas consequências, estabelecendo relações entre $\mathrm{MoC}$ e a gestão da inovação. Nessa perspectiva, Poole e Van De Ven (2004, p. 375) apresentam uma tipologia das teorias de mudança organizacional e inovação, sugerindo que modelos simples podem ser combinados para gerar teorias mais complexas.

Segundo Poole e Van De Ven (2004, p. 375), as teorias de mudança organizacional e inovação tendem a ser complexas, geralmente combinando muitos mecanismos geradores diferentes. Tais autores apresentam uma fundação para o estudo de tal temática com quatro possibilidades fundamentais, sendo que cada uma teria um diferente motor gerador direcionando o processo de mudança: (1) teoria do ciclo de vida, (2) teoria da teleologia, (3) teoria dialética e (4) teoria da evolução. Em situações práticas, sempre duas ou mais dessas quatro teorias operariam em conjunto em diferentes níveis ou em diferentes períodos de tempo. As teorias podem ser discutidas em termos de: (i) previsão ou não do estado final do processo; (ii) predeterminação ou não da trajetória de desenvolvimento; (iii) convergência ou divergência do processo; e (iv) se o tempo se baseia em eventos ou em ciclos. As quatro teorias são brevemente apresentadas a seguir.

A teoria do ciclo de vida (POOLE; VAN DE VEN, 2004, p. 376) se define pelo progresso da mudança através de uma sequência de estágios ou fases conforme prescrito ou regulado por um programa institucional, lógico ou natural definido no início do ciclo. O motor da mudança advém de uma forma ou padrão inerente à entidade em transição ou imposta por uma instituição externa via regras e regulamentações. Tão logo se atinja o final do ciclo, um novo processo se inicia com a mesma ou com outra entidade.

A teoria da teleologia (POOLE; VAN DE VEN, 2004, p. 378), ou da transição intencional, percebe a transição como um ciclo de formulação de objetivo, implementação, avaliação e modificação de ações ou objetivos com base no que foi aprendido ou pretendido pela entidade. Tal sequência surge da construção social de um estado final pretendido pelos indivíduos da entidade em transição. Desse modo, definir um objetivo em resposta a um problema percebido ou a uma oportunidade coloca o processo em andamento. Dado o direcionamento a objetivos, múltiplos caminhos são possíveis, fato que não permite a definição prévia de estágios ou passos a serem seguidos.

A teoria dialética (POOLE; VAN DE VEN, 2004, p. 379), ou da transição por conflito, se refere à mudança originada pelos esforços de se lidar com contradições, conflitos 
ou tensões dentro ou fora da entidade em transição. Nesse caso, o objetivo ou o ponto final do processo de mudança não está claro no começo, emergindo desse processo dialético. Por conseguinte, a reação aos acontecimentos gera caminhos com passos que variam enormemente a cada caso.

A teoria da evolução (POOLE; VAN DE VEN, 2004, p. 380), ou mudança pela competitividade, consiste de uma sequência repetitiva de eventos de variação, seleção e retenção entre entidades de uma certa população. O contexto é o da competição por recursos ambientais escassos entre as entidades que integram uma determinada população. Assim, os passos durante a seleção e a retenção não podem ser planejados ao longo do tempo devido às mudanças nas pressões competitivas pelos recursos, fato que gera caminhos com passos fracamente predeterminados, com um ou mais ciclos de variação, seleção e retenção com atividades indeterminadas através desses ciclos.

Por certo, a maioria dos processos de mudança organizacional e inovação observados são mais complexos que as quatro teorias discutidas: (1) ciclo de vida, (2) teleologia, (3) dialética e (4) evolução. Por conseguinte, mais de um motor de mudança pode atuar em um caso particular. Assim sendo, Poole e Van De Ven (2004, p. 382) discutem de maneira aprofundada a interação entre diferentes motores, explicitando como gerar teorias compostas pela combinação de motores e partindo da perspectiva de que as unidades de uma organização estão situadas em um sistema de vários níveis, com indivíduos em departamentos dentro das organizações que, por sua vez, integram indústrias de um setor ou comunidades dentro de nações ou culturas. Mais ainda, há situações em que as unidades que sofrem a transição se aninham em hierarquias que tornam a mudança dependente também das unidades de níveis mais baixos ou mais altos, cenário que demanda maior complexidade dos modelos a serem utilizados.

No caso da UNICAMP, percebem-se características da solução conhecida por teoria do ciclo de vida, como discutido acima, que se define pelo progresso da mudança através de uma sequência de estágios ou fases conforme prescrito ou regulado por um programa institucional, lógico ou natural definido no início do ciclo, com o motor da mudança sendo uma forma, um padrão, um conjunto de regras ou um conjunto de regulamentações. Assim, na UNICAMP, percebemos uma busca pela institucionalização dos métodos e práticas afins ao BSC no PLANES-UNICAMP, como indicado a seguir. 
A UNICAMP utiliza o BSC, com o estabelecimento de objetivos estratégicos para os quais se definem indicadores que terão metas específicas que possam ser verificadas. Segundo o relatório "Gestão Estratégica: Planejamento Estratégico e Avaliação Institucional" (UNICAMP, 2012, p. 4), fica evidenciado que "ainda são muito incipientes os trabalhos de aplicação do BSC a Instituições de Ensino Superior e, mais ainda, a Instituições com as características da UNICAMP”. São cinco as áreas estratégicas contempladas no PLANESUNICAMP: (1) ensino; (2) pesquisa; (3) extensão; (4) administração; e (5) qualidade de vida. Para cada área estratégica são definidas estratégias, programas e linhas de ação, sendo que os programas são implantados através de projetos estratégicos. Tais projetos são revistos anualmente pela Comissão de Planejamento Estratégico Institucional, ou COPEI-UNICAMP, da qual o primeiro autor deste texto teve a oportunidade de participar como membro titular em várias ocasiões nos últimos anos.

Para apoiar os processos de avaliação institucional e planejamento estratégico, a UNICAMP faz uso do SIPLANES, um sistema de informação baseado no software MediaWiki (http://www.mediawiki.org/wiki/MediaWiki), o qual é gratuito e de código aberto. O software foi escrito em PHP (http://www.php.net/), uma linguagem de propósito geral amplamente utilizada e que é especialmente adequada para o desenvolvimento Web, sendo passível de incorporação de HTML (http://www.w3.org/MarkUp/). O software MediaWiki foi originalmente concebido tendo como foco a enciclopédia livre WikiPedia (http://www.wikipedia.org/). A palavra "Wiki", de maneira geral, indica um ambiente Web colaborativo que pode ser editado por usuários autorizados.

No caso da UNICAMP, o sistema de informação SIPLANES (http://siplanes.unicamp.br/) tem acesso restrito com autenticação por senha. A navegação por páginas segue o agrupamento destacado pela seguinte indexação: página principal; revisão do PLANES 2011-2015; ambiente de avaliação interna; ambiente de avaliação externa; glossário; banco de dados; quadro de avisos; mudanças recentes; eventos atuais; e ajuda. Tal sistema de informação foi implantado para a avaliação institucional do período 2004-2008, sendo utilizado pelos membros das comissões de avaliação interna e externa. Com isso, foi possível a elaboração colaborativa de documentos afins às análises a partir da redação de respostas a questionários. A autenticação por senha garantiu segurança mesmo com o acesso via internet, sendo que o acesso atual fica restrito aos membros das comissões de avaliação e 
aos comitês das unidades, colégios, centros e núcleos interdisciplinares responsáveis pela revisão dos planejamentos estratégicos locais.

Conforme se discute em detalhe na seção que segue, fica evidente que, em casos como o da UNICAMP, temos sistemas de informação já bastante avançados, mas que ainda não contemplam ferramentas e técnicas que caracterizam uma cultura orientada a "analytics", com o melhor uso de dados de fontes diversas.

\section{BUSCA PELA INOVAÇÃO ATRAVÉS DE NOVOS MODELOS GERENCIAIS}

A seção anterior apresentou, em linhas gerais, o planejamento estratégico da UNICAMP, uma das poucas universidades brasileiras a ter tal estruturação, fato que tornou tal universidade uma referência no assunto dentro e fora do Brasil. Pode-se perceber que, em casos como o da UNICAMP, temos sistemas de informação que não contemplam funcionalidades que caracterizem uma cultura orientada a "analytics".

Uma abordagem de "Information and Communication Technology" (ICT) que priorize "analytics" pode viabilizar o uso de dados e de introspecções associadas a eles com o apoio de disciplinas como estatística, ciências cognitivas, matemática aplicada e outras, tendo nesse caso a computação como pano de fundo para que se automatizem parcial ou totalmente muitos dos processos rotineiros e repetitivos. Conforme se discutiu em seções anteriores, tais ferramentas e técnicas poderiam vir a permitir que as universidades se baseassem de maneira mais abrangente e eficaz em fatos para atividades desde o planejamento até as decisões, passando pela execução, pelo gerenciamento e pela medição, incluindo, por fim, a aprendizagem organizacional.

Mais ainda, em uma cultura orientada a "analytics", ferramentas e técnicas para o que se chama de "big data" podem vir a ser de interesse, o que nesse caso inclui novos modos de agregar, manipular, analisar e visualizar grandes volumes de dados em uma abordagem flexível e multidisciplinar. Isso permitiria melhor integrar dados hoje dispersos e nem sempre utilizados, em especial os que se referem às comunidades interna e externa que interagem de algum modo com a universidade: alunos, docentes, funcionários, empresas públicas e privadas, governos etc.

Exemplos de informações de interesse que poderiam comparecer em "dashboards" e/ou compor indicadores incluem estatísticas sobre a quantidade de alunos da universidade que fazem estágios no Brasil e no exterior, assim como sobre a quantidade de estrangeiros que 
participam de atividades diversas em várias unidades universitárias. A importância de indicadores associados é evidente nesse caso específico, mesmo porque a internacionalização é uma das prioridades de tal instituição.

Como bem se evidencia em seções anteriores, o planejamento estratégico ainda é algo recente em universidades, em especial caso se considere universidades como a UNICAMP, as quais se beneficiam de soluções como o BSC mas que ainda não fazem uso das novas possibilidades de um OSM e/ou de "analytics", por exemplo.

Nesse sentido, é de interesse que se identifiquem as características de um novo sistema de informação que vá além do SIPLANES. Tal sistema de informação deve se basear em algum modelo de referência que seja apropriado ao contexto das universidades. Na próxima seção é apresentada uma visão geral de como poderia ser um modelo com tais características.

\section{PROPOSTA DE MODELO PARA UNIVERSIDADES}

Modelos de referência para a gestão podem se beneficiar do uso das "Information and Communication Technologies", sendo a tecnologia uma das cinco abordagens mais comuns para promover mudanças fundamentais. Langley et al. (2009, p. 133) explicitam quais seriam essas abordagens: (1) pensamento lógico sobre o sistema atual; (2) benchmarking e aprendizado adquirido com outras pessoas; (3) uso de tecnologia; (4) pensamento criativo; e (5) uso de conceitos de mudança.

A abordagem relativa ao pensamento lógico sobre o sistema atual (LANGLEY et al., 2009, p. 133) se refere a, inicialmente, documentar a maneira como os processos são executados ou como um produto funciona. A coleta e a análise de dados contribuem para a identificação das causas de problemas no sistema atual, o que depois sugerirá quais mudanças seriam as mais apropriadas. Isso pode ser feito por amostragem e/ou através de estudos de caso, sendo que em alguns casos podemos fazer uso de análise estatística com simulações, mineração de dados, análise de regressão etc.

A abordagem relativa ao benchmarking e aprendizado adquirido com outras pessoas (LANGLEY et al., 2009, p. 136) se refere a buscar adaptar melhorias percebidas em outras organizações, em especial com o foco em boas práticas que resultam em alto nível de desempenho. Nesse sentido, são feitas observações para que depois as informações sejam utilizadas na busca de melhorias. Para tanto, é essencial não apenas buscar documentar e 
copiar, mas sim compreender os mecanismos causais que indiquem qual a razão de algo funcionar bem.

A abordagem relativa ao uso de tecnologia (LANGLEY et al., 2009, p. 139) se refere a utilizar não apenas sistemas de informação, mas também a fazer uso prático da ciência através de equipamentos, materiais e métodos. No caso específico dos sistemas de informação, as vantagens incluiriam desde redução de custos até melhoria de qualidade, além da eventual criação de novos produtos e serviços. Para se reduzir os riscos de implementação, as tecnologias de interesse devem ser testadas em pequena escala e com apoio de um plano de gerenciamento de transição que auxilie as pessoas na adaptação ao novo contexto.

A abordagem relativa ao pensamento criativo (LANGLEY et al., 2009, p. 142) se refere à invenção de novas ideias para promover mudanças fundamentais, nesse caso buscando-se selecionar e refinar propostas que possam resultar em melhorias. O pensamento criativo pode se beneficiar de novas formas de pensar, sendo inúmeros os métodos provocadores.

A abordagem relativa ao uso de conceitos de mudança (LANGLEY et al., 2009, p. 146) se refere ao uso de conceitos ou abordagens específicas, como as seguintes: usar medidas apropriadas, promover treinamento, usar amostragem, alterar a ordem das etapas de um processo, padronizar criando processos formais, dar acesso à informação, utilizar automação etc. Tal uso de conceitos ou abordagens específicas tende a provocar novas formas de pensar.

Um modelo de referência que seja apropriado ao contexto das universidades foi desenvolvido com base no pensamento lógico sobre o sistema atual e na perspectiva do uso de tecnologia, em especial no que se refere a dar mais acesso à informação e a utilizar automação parcial ou total. Como é evidente, cuidados no uso de tecnologia (LANGLEY et al., 2009, p. 140) envolvem não automatizar um sistema ruim. Nesse sentido, deve-se buscar redesenhar e melhorar o sistema antes de automatizá-lo.

Nessa perspectiva, o framework "WCU Office of Strategy, Analytics and Continuous Improvement" (WCU-OSACI), discutido a seguir, teria (1) um ciclo baseado nos conceitos de OSM e de BSC prevendo a integração de subsistemas afins aos macroprocessos de execução da estratégia, (2) um modelo de maturidade voltado à melhoria contínua de processos gerenciais com foco em uso intensivo de sistemas de informação via automação e, por fim, (3) uma implementação baseada no uso de um ou mais softwares de suporte. Tal proposta de framework é apresentada em maiores detalhes a seguir. 
O framework WCU-OSACI teria um ciclo baseado nos conceitos de OSM e de BSC prevendo a integração de subsistemas afins aos macroprocessos de execução da estratégia. $\mathrm{O}$ ciclo, desse modo, teria os passos tradicionais: (1) desenvolvimento da estratégia; (2) planejamento da estratégia; (3) alinhamento da organização; (4) planejamento das operações; (5) monitoramento e aprendizado; e (6) teste e adaptação. Nesse sentido, o OSM seria arquiteto da gestão estratégica, seria dono de vários processos e seria integrador. Como arquiteto, o OSM definiria a estrutura e as normas da gestão estratégica e projetaria tal gestão estratégica. Como dono dos processos concernentes, desenvolveria e planejaria a estratégia, alinharia a organização e avaliaria a estratégia, realizando adaptações quando necessário. Como integrador, integraria planejamento e orçamentação operacional, integraria processos operacionais essenciais, integraria recursos humanos com tecnologia da informação e funções de apoio, comunicaria a estratégia, gerenciaria as iniciativas estratégicas e compartilharia as melhores práticas.

O framework WCU-OSACI teria oito níveis de maturidade em busca da melhoria contínua dos processos: (1) processo incomum, que quase nunca ocorre e dificilmente seria padronizado; (2) processo espontâneo, que poderia ser padronizado, por ser frequente; (3) processo padronizado; (4) processo mensurável; (5) processo controlável; (6) processo aprimorável; (7) processo automatizável parcialmente; e (8) processo automatizável totalmente. Note-se, portanto, que teríamos o dobro da quantidade de níveis de maturidade tradicional, com os quatro níveis "standardize", "measure", "control" e "improve". Ter mais níveis permite uma avaliação mais cuidadosa. Em especial, cabe destacar o foco em automação parcial ou total nos dois últimos níveis propostos após uma busca preliminar de aprimoramento.

Como se evidenciou ao longo do texto, o BSC sugere os processos internos prioritários. A partir daí, a maturidade dos processos selecionados passa a ser acompanhada com uma análise em oito níveis. Ao longo do tempo, a produtividade dos funcionários aumentaria, inclusive pela menor intervenção humana em uma perspectiva de automação após aprimoramento dos processos. Depois de se conseguir uma melhoria da qualidade e da produtividade, viriam considerações adicionais sobre cortes de custos - sempre que possível, com diminuição dos prazos tradicionais de execução dos processos. No momento de aprimorar, devemos definir o que e/ou qual característica do processo se pretende aprimorar, como facilidade de compreensão do processo, confiabilidade, robustez etc. Por fim, o 
aprimoramento deve ser similar ao "Capability Maturity Model Integration" (CMMI http://www.sei.cmu.edu/cmmi/) contínuo, considerando a maturidade em cada um dos processos prioritários para que, assim, seja possível comparar como cada unidade da organização lida com um mesmo processo comum às várias unidades. Tal comparação permitiria, desse modo, indicar a maturidade por unidade da organização.

O framework WCU-OSACI teria uma implementação baseada no uso de um ou mais softwares de suporte, sendo possível tanto orquestrar o uso de vários softwares como também desenvolver um novo sistema que abarcasse toda a complexidade inerente de maneira integrada. Nesse último caso, a identificação dos requisitos mínimos sugeriria prototipagem com as fases tradicionais (O’BRIEN, 2010, p. 327): (1) identificação de requisitos de informação dos usuários finais, com investigação e análise; (2) desenvolvimento de protótipo do sistema de informação, com análise e projeto; (3) revisão do protótipo para atender melhor aos requisitos do usuário final, com projeto e implantação; e (4) utilização e manutenção do sistema aceito, com implantação e manutenção ao longo do ciclo de vida. A prototipagem irá demandar (a) definir uma equipe, (b) gerar um esquema a ser convertido em protótipo, (c) apresentar tal esquema e/ou protótipo aos usuários, (d) receber feedback dos usuários após tal apresentação, (e) realizar novos ciclos de apresentações com busca de feedback adicional, (f) realizar reuniões com outras partes interessadas na busca tanto de melhorias adicionais como de conformidade com padrões em uso na organização, (g) buscar aprovação final do novo sistema desenvolvido e (h) instalar o novo sistema desenvolvido.

Vale notar que, em uma cultura orientada a "analytics", ferramentas e técnicas para o que se chama de "big data" podem vir a ser de interesse, como discutido em seção anterior. Isso permitiria novos modos de agregar, manipular, analisar e visualizar grandes volumes de dados com ferramentas e técnicas que incluiriam "crowdsourcing", "predictive modeling", "simulation" e "visualization".

Esta investigação prosseguirá futuramente com um maior detalhamento do framework WCU-OSACI assim como com uma especificação detalhada do novo sistema a ser desenvolvido.

\section{TENDÊNCIAS FUTURAS}

Tendências futuras incluem considerações sobre o uso crescente de interfaces multimodais em equipamentos diversos, a ubiquidade afim ao barateamento e à melhoria dos 
dispositivos e a mobilidade com dispositivos que podem ser transportados facilmente. Isso sugere a geração de uma quantidade massiva de dados que podem ser integrados com uso de ferramentas e técnicas de "information fusion" para posterior manipulação e tratamento com estratégias de "analytics", muitas das quais com base nas ciências matemáticas.

Neste texto, a multimodalidade (W3C, 2003, p. 1) se refere aos vários modos de interação, com combinações de fala, gestos, texto, imagem etc. para interagir com os computadores. Os tipos de interface já são muitos, crescem a cada dia e podem incluir funcionalidades para detecção de emoções, reconhecimento de humor, computação afetiva, "human augmentation", "wearable computers", rastreamento ocular, análise de dados sobre o comportamento do usuário, reconhecimento de gestos, sensações através da pele e/ou do toque, processamento de linguagem natural, reconhecimento da voz e controle de equipamentos pela fala, biometria, bioacústica, dentre outras possibilidades.

A mobilidade (SAP, 2011, p. 4) se refere a se mover com as máquinas, o que neste caso sugere o uso de dispositivos móveis que podem ter internet, com ubiquidade, e podem oferecer experiências de realidade aumentada, com sobreposição de informações sobre uma imagem captada pela câmera do aparelho. Com ubiquidade (W3C, 2012a, p. 1), torna-se tanto possível como relevante às pessoas acessar os sistemas de informação de interesse sempre que se necessitar de informações, o que por sua vez torna essencial a atualização de tais sistemas em tempo real.

A mobilidade (GARTNER, 2011, p. 6) se insere no contexto do crescente acesso a dispositivos móveis, o que inclui desde tablets até smartphones, paralelamente à utilização de laptops e outros tipos de aparelhos. O uso de diferentes tipos de dispositivos tanto para o acesso à informação como para a captura de dados torna essencial considerar como melhor selecionar as informações capturadas, agregando-as e manipulando-as de maneira padronizada, como também torna importante considerar como melhor apresentar as informações em diferentes tipos de dispositivos e para diferentes partes interessadas (W3C, 2012b, p. 1).

Para ser possível uma posterior manipulação e um posterior tratamento de dados com "analytics", se faz necessário realizar a fusão de dados e de informações. Nesse sentido, funcionalidades de "information fusion" devem passar a estar presentes em sistemas de informação como os que são tratados neste texto, inclusive com processamento em tempo real em bancos de dados distribuídos (US, 2012, p. 1). Por "information fusion" se entende a 
integração de informação e de dados de diferentes fontes relativamente ao comportamento de um sistema em particular. Tais dados e informações, quando apropriadamente utilizados através de diferentes ferramentas e técnicas, dão suporte a ações e decisões afins ao sistema de interesse. As fontes de dados e informações são múltiplas, incluindo sensores que observam o comportamento do sistema, bancos de dados com conhecimento relevante sobre comportamento prévio, informações coletadas por seres humanos e simulações prevendo comportamento futuro do sistema. A combinação de dados puros de diferentes fontes com informações disponíveis tente a permitir um melhor entendimento de vários fenômenos de interesse para a tomada de decisão na perspectiva da gestão estratégica, subsidiando assim as partes interessadas que fizerem uso do sistema de informação gerencial de suporte. Nesse sentido, é essencial fazer uso de métodos para transformar os dados e as informações, para identificar as fontes potenciais de dados e de informações, para automatizar a fusão de dados e de informações, para perceber os efeitos de determinados dados e informações em diferentes situações relacionadas à tomada de decisão e para melhor desenvolver sistemas de informação que façam uso de fusão de dados.

\section{CONCLUSÃO}

Este trabalho objetivou discutir a importância da utilização de tecnologias de informação e de comunicação em WCUs, considerando em especial o planejamento estratégico. Foram discutidas questões de interesse para universidades do Brasil e do resto do mundo motivadas a se inserir no seleto grupo das universidades de elite, as quais competem para prover a sociedade com conhecimento, com pesquisa e com iniciativas diversas que promovam ganhos qualitativos no bem-estar social. O texto apresentou os procedimentos utilizados por uma universidade brasileira para realizar seu planejamento estratégico, inclusive discutindo aspectos relativos à utilização de sistemas de informação. Também se discutiu como melhor utilizar soluções baseadas em "analytics" para lidar com dados e informações disponíveis, assim como se discutiu como automatizar processos após a realização de melhorias neles.

Foi também apresentado e discutido o framework "WCU Office of Strategy, Analytics and Continuous Improvement" (WCU-OSACI). Tal framework seria composto de três partes essenciais. A primeira se referiria a um ciclo baseado nos conceitos de OSM e de BSC prevendo a integração de subsistemas afins aos macroprocessos de execução da estratégia. A 
segunda parte se referiria a um modelo de maturidade voltado à melhoria contínua de processos gerenciais com foco em uso intensivo de sistemas de informação via automação. Por fim, a terceira parte estaria relacionada a uma implementação baseada no uso de um ou mais softwares de suporte.

Trabalhos futuros apresentarão tanto um maior detalhamento do framework WCUOSACI quanto uma especificação detalhada do novo sistema a ser desenvolvido. Para tanto, tendências futuras serão investigadas mais aprofundadamente, o que sugere a inclusão de considerações sobre multimodalidade, ubiquidade e mobilidade.

\section{REFERÊNCIAS}

BARBIERI, J. Idealizador do Planes defende tese de doutorado na FEEC. Portal UNICAMP. Universidade Estadual de Campinas, 29 abr. 2008. Disponível em:

$<$ http://www.unicamp.br/unicamp/divulgacao/2008/04/30/idealizador-do-planes-defende-tesede-doutorado-na-feec $>$. Acesso em: 12 jun. 2012.

CERTO, S. C.; PETER, J. P. Administração estratégica: planejamento e implantação da estratégia. São Paulo: Prentice Hall, 2005. 320 p.

GARDENAL, I. Planes da UNICAMP caminha rumo à sua consolidação. Portal UNICAMP. 21 ago. 2008. Disponível em:

$<$ http://www.unicamp.br/unicamp/divulgacao/2008/08/21/planes-da-unicamp-caminha-rumoa-sua-consolidacao $>$. Acesso em: 12 jun. 2012.

GARTNER. iPad and beyond: what the future of computing holds. Gartner, Inc., 30 set. 2011. Disponível em: <http://www.gartner.com/id=1812319>. Acesso em: 12 jun. 2012.

GORDON, R. A.; HOWELL, J. E. Higher education for Business. New York: Columbia University Press, 1959. 494 p. Disponível em: <http://www.questia.com/library/book/highereducation-for-business-by-robert-aaron-gordon-james-edwin-howell.jsp $>$. Acesso em: 12 jun. 2012.

HARRINGTON, H. J.; CONNER, D.; HORNEY, N. L. Project change management. New York, NY: McGraw-Hill Companies. 16 dez. 1999. 332 p.

KAPLAN, R. S.; NORTON, D. P. A estratégia em ação: Balanced Scorecard. Rio de Janeiro: Campus Elsevier, 1997.

KAPLAN, R. S.; NORTON, D. P. The execution premium: linking strategy to operations for competitive advantage. Boston, MA: Harvard Press, 2008.

KIECHEL, W. Os mestres da estratégia. Rio de Janeiro, RJ: Campus, 2011. 320p. 
KIRON, D. et al. Analytics: the widening divide - how companies are achieving competitive advantage through analytic. IBM \& MIT Sloan Management Review, out. 2011. Disponível em: <http://www-142.ibm.com/>. Acesso em: 12 jun. 2012.

KOTTER, J. P. Leading change. Boston, MA: Harvard Business School Press, 1996. 208 p.

LANGLEY, G. J. et al. The improvement guide: a practical approach to enhancing organizational performance. San Francisco: Jossey-Bass Business \& Management, 2009.

MGI - McKinsey Global Institute. Big data: The next frontier for innovation, competition, and productivity. Boston, MA: McKinsey \& Company, jun. 2011. Disponível em:

$<$ http://www.mckinsey.com/Insights/MGI/Research/Technology_and_Innovation/Big_data_T he_next_frontier_for_innovation>. Acesso em: 12 jun. 2012.

MINTZBERG, H.; AHLSTRAND, B.; LAMPEL, J. Safári de estratégia. Porto Alegre, RS: Bookman, 2010. 392p.

O'BRIEN, J. A. Sistemas de informação e as decisões gerenciais na era da internet. São Paulo: Saraiva, 2010.

POOLE, M. S.; VAN DE VEN, A. H. Theories of organizational change and innovation processes. In: POOLE, M. S.; VAN DE VEN, A. H. (Orgs.). Handbook of organizational change and innovation. New York: Oxford University Press, 2004. 456 p.

SAP. Mobility, its impact, opportunities, and challenges: gaining a business perspective on mobility. SAP Executive Insight, Mobility Series, 2011. Disponível em:

$<$ http://www.sap.com/campaigns/2011_04_mobility/assets/Mobility-

Its_Impact_Opportunities_and_Challenges.pdf $>$. Acesso em: 12 jun. 2012.

SEBRAE. Fatores condicionantes e taxa de mortalidade de empresas no Brasil. Relatório de pesquisa. Serviço de Apoio às Micro e Pequenas Empresas de Minas Gerais -

SEBRAE/MG. Nov. 2004. Disponível em:

$<$ http://www.sebraemg.com.br/arquivos/informativos/relatorio_pesquisa_mortalidade_minas. pdf $>$. Acesso em: 12 jun. 2012.

SHOEMAKER, P. J. H. Scenario planning: a tool for strategic thinking. Sloan Management Review. Boston, MA: MIT. 1995.

THE GORDON-HOWELL report of 1959: A seminal critique of American business education, five decades on. The Economist, London, 4 jun. 2009. Disponível em: $<$ http://www.economist.com/node/12762453>. Acesso em: 12 jun. 2012.

UNICAMP. Coordenadoria Geral da Universidade (CGU). Planejamento Estratégico (20072010): Estrutura do Plano Estratégico. Campinas, 2008. Disponível em: $<$ http://siplanes.unicamp.br/planes/PlanejamentoEstrategico/AprovadoCONSU2008.pdf $>$. Acesso em: 12 jun. 2012. 
Coordenadoria Geral da Universidade (CGU). Gestão estratégica: planejamento estratégico e avaliação institucional. Campinas, 2012. Disponível em:

$<$ http://siplanes.unicamp.br/planes/PlanejamentoEstrategico/MapaEstrategico.pdf $>$. Acesso em: 12 jun. 2012.

US. Infrastructure for information fusion (StrucFus). Information Fusion Research Program. University of Skövde (US), 2012. Disponível em:

$<$ http://www.his.se/english/research/infofusion/research/associated-projects/infrastructure-forinformation-fusion-strucfus/>. Acesso em: 12 jun. 2012.

W3C - World Wide Web Consortium. Multimodal interaction framework. W3C NOTE 06 May 2003. Disponível em: <http://www.w3.org/TR/mmi-framework/>. Acesso em: 12 jun. 2012.

Ubiquitous Web. 2012a. Disponível em: <http://www.w3.org/UbiWeb/>. Acesso em: 12 jun. 2012.

Web accessibility initiative. 2012b. Disponível em: <http://www.w3.org/WAI/>. Acesso em: 12 jun. 2012. 